\title{
Avaliação da acurácia de dois glicosímetros veterinários para uso em cães
}

\section{Accuracy evaluation of two veterinary glucometers for use in dogs}

\author{
Maria Lopes Correa ${ }^{1}$, Fabiola de Oliveira Paes Leme ${ }^{2}$, Adriane Pimenta da Costa Val ${ }^{3}$
}

\section{Resumo}

Os glicosímetros (GT) são estratégia importante para mensuração da glicemia. Devem estar dentro das especificações da ISO 15197:2013 e avaliados pela análise da grade de erros. Objetivou-se avaliar a acurácia de dois glicosímetros veterinários (GT1 e GT2), pela comparação dos valores de glicemia mensurados em sangue capilar, com aqueles fornecidos por métodos laboratoriais de referência em amostras plasmáticas, transformadas ou não em valores de plasma equivalente. Na avaliação de GT1 em amostras de sangue capilar e o método laboratorial hexoquinase obtiveram-se média das diferenças de $25,90 \mathrm{mg} / \mathrm{dL}$ e $26 \%$ das amostras recaíram sobre os limites propostos pela ISO15197: 2013. A comparação de GT1 aos valores de glicemia estimados para plasma equivalente, mostrou média de diferenças de $10,89 \mathrm{mg} / \mathrm{dL}$ e $99 \%$ das amostras atenderam aos limites pretendidos. Comparações avaliação de GT2 e o método laboratorial glicose oxidase em amostras de sangue total capilar, a média das diferenças foi $10,58 \mathrm{mg} / \mathrm{dL}$ e $22 \%$ das amostras atenderam aos intervalos propostos pela ISO15197: 2013; quando avaliado pela glicemia estimada para plasma equivalente, a média das diferenças foi de $9,7029 \mathrm{mg} / \mathrm{dL}$ e $99 \%$ das amostras atenderam aos limites. Quando avaliados pela grade de erros de erros, $87 \%$ das amostras avaliadas por GT1 recaíram sobre as zonas de erros A e B e aquelas avaliadas por GT2 apresentaram $86 \%$ das amostras sobre tais zonas. Os glicosímetros avaliados não apresentaram a acurácia para mensuração da glicemia capilar conforme os padrões expostos pela normativa ISO 15197:2013.

Palavras chave: Glicemia. Monitoramento. Ensaios clínicos.

\begin{abstract}
The glucometers (GT) are important strategy for measuring blood glucose. They must be within the specifications of ISO 15197: 2013 and evaluated by analysis of the error grid. The objective of this study was to evaluate the accuracy of two veterinary glucometers (GT1 and GT2) by comparing the blood glucose values measured in capillary blood with those provided by reference laboratory method in plasma samples, transformed or not at equivalent plasma values. In the evaluation of GT1 in capillary blood samples and the hexokinase laboratory method, the mean differences were $25.90 \mathrm{mg} / \mathrm{dL}$ and $26 \%$ of the samples fell within the limits proposed by ISO 15197: 2013. The comparison of GT1 to the estimated plasma glucose values showed a mean difference of $10.89 \mathrm{mg} / \mathrm{dL}$ and $99 \%$ of the samples were in the desired limits. Comparisons of GT2 and laboratory glucose oxidase in capillary blood samples, mean differences were $10.58 \mathrm{mg} / \mathrm{dL}$ and $22 \%$ of the samples met the ranges proposed by ISO; 15197: 2013. When evaluated by the estimated plasma glucose, the mean differences were 9.7029 $\mathrm{mg} / \mathrm{dL}$ and $99 \%$ of the samples met the limits. When evaluated by the error grid, $87 \%$ of the samples evaluated by GT1 fell on the zones of errors A and B and those evaluated by GT2 presented $86 \%$ of the samples on such zones. The glucometers evaluated did not present the accuracy to measure capillary glycaemia according to the standards set in ISO 15197: 2013.
\end{abstract}

Keywords: Blood glucose. Monitoring. Clinical trial.

\footnotetext{
${ }^{1}$ Mestranda em Ciência Animal pela Escola de Veterinária da Universidade Federal de Minas Gerais, Belo Horizonte, Minas Gerais, Brasil

${ }^{2}$ Doutorado em Ciência Animal pela Universidade Federal de Minas Gerais, Belo Horizonte, Brasil. Professora associada I da Escola de Veterinária da Universidade Federal de Minas Gerais, Belo Horizonte, Brasil.

${ }^{3}$ Doutorado em Ciência Animal pela Universidade Federal de Minas Gerais, Belo Horizonte, Minas Gerais. Professora Associada da II Escola de Veterinária da Universidade Federal de Minas Gerais, Departamento de Clínica e Cirurgia Veterinária, Belo Horizonte, Minas Gerais, Brasil. E-mail: adriane.costaval@gmail.com
} 


\section{Introdução}

Glicosímetros (GT) são aparelhos que quantificam a concentração de glicose no sangue por meio de reação eletro-enzimática. ${ }^{(1,2)}$ São ferramentas acessíveis devido à simplicidade da técnica de manuseio, que requer pouco treinamento, e tampouco preparo do paciente para a coleta das amostras. São empregados na rotina médico veterinária em diversas situações marcadas por disglicemias acentuadas, possibilitando a inferência acerca de sua integridade metabólica e contribuindo para a adequação das condutas terapêuticas, permitindo, assim, o estabelecimento de prognóstico assertivo. ${ }^{(2-4)}$

Deste modo, é fundamental que os aparelhos adotados sejam confiáveis, principalmente para avaliarem amostras coletadas de pacientes em diferentes graus de comprometimento da integridade metabólica e de saúde geral.(4-6) O mercado tem oferecido GT desenvolvidos para uso exclusivo na assistência veterinária. É esperado que tais aparelhos sejam programados para processarem amostras capilares das espécies domésticas a que se propõe, incluindo as possíveis variações hematológicas. ${ }^{(4,7)}$

No intuito de ampliar a padronização dos GT, diferentes organizações especializadas têm apresentado diretrizes definindo padrões de variação e de avaliação de tais sistemas. Na medicina veterinária, a American Society for Veterinary Clinical Pathology apresentou suas diretrizes para avaliação de GT e sua confirmação de credibilidade para uso na clínica de cães e gatos, com base em estratégias de controle de qualidade e melhoria de confiabilidade. ${ }^{(8)}$ Os estudos de referência da área frequentemente utilizam parâmetros definidos pela International Organization for Standadization, que, sob a inscrição ISO 15197:2013, sugere critérios para avaliação dos GT disponíveis no mercado. Tal normativa propõe intervalos de variação aceitáveis para os valores de glicemia fornecidos pelos GT em comparação aos obtidos por método laboratorial de referência (MLab) que represente a real condição glicêmica in vivo. ${ }^{(7,9,10)}$

Os critérios apresentados pela ISO 15197:2013 estabelecem dois intervalos de variação dentro dos quais devem estar ao menos $95 \%$ dos resultados de glicemia obtidos nos GT avaliados. ${ }^{(1)}$ Tais intervalos de variação são diferentes para cada amostra, pois são estabelecidos a partir do valor de glicemia obtido pelo MLab. Sendo assim, quando o MLab mensurar concentração de glicose inferior a $100 \mathrm{mg} / \mathrm{dL}$, o valor da glicemia fornecido pelo GT avaliado pode variar em \pm $15 \mathrm{mg} / \mathrm{dL}$ em relação ao primeiro. Por sua vez, quando o MLab identifica glicemia a partir de $100 \mathrm{mg} / \mathrm{dL}$, o GT avaliado pode fornecer valores que difiram $\pm 15 \%$. Além disso, a acurácia do aparelho deve ser avaliada pela grade de análises de erros (GAE) proposta por Parkes et al. ${ }^{(12)}$, metodologia que categoriza os erros de mensuração em zonas de acordo com o possível impacto clínico que tais erros possam acarretar sobre a saúde de pacientes com diabetes tipo I. Ao menos 99\% de dosagens glicêmicas feitas pelo GT testado devem estar nas zonas A e B, que representam menor impacto de mensurações equivocadas sobre a conduta terapêutica. ${ }^{(7,13)}$

Estudos que colaborem para a definição das limitações de uso dos GT podem contribuir para a redução da ocorrência de erros de conduta clínica veterinária decorrentes de mensurações glicêmicas equivocadas. Portanto, o objetivo desse trabalho foi avaliar a acurácia de dois GT veterinários para uso na espécie canina de acordo com o parâmetro de acurácia proposto pela normativa ISO 15197:2013 e pela análise da grade de erros.

\section{Material e Métodos}

O estudo foi conduzido do ano de 2016, entre os meses de maio e agosto, nas dependências hospital veterinário de pequenos animais da Escola de Veterinária da Universidade Federal de Minas Gerais (EV/UFMG). O projeto foi aprovado pelo comitê de ética em experimentação animal da mesma instituição (CEUA/UFMG), sob a inscrição no 383/2015.

A participação de cada paciente no estudo foi autorizada mediante assinatura de Termo de Consentimento Livre e Esclarecido por seus responsáveis. Todos os pacientes foram submetidos à avaliação clínica geral previamente a qualquer procedimento do estudo. Para a inclusão deveriam ser da espécie canina, ter idade superior a seis meses e apresentarem adequada perfusão capilar. Além disso, foram estabelecidos critérios para inclusão das $\operatorname{amostras}^{(13)}$ com base nas informações fornecidas pelos fabricantes dos GT avaliados nas respectivas bulas: glicemia capilar mensurada por ambos GT e por dois MLab; glicemia venosa mensurada pelo MLab entre 20 e $600 \mathrm{mg} / \mathrm{dL}$; hematócrito superior a 20\%; ausência de lipemia intensa.

O estudo avaliou a acurácia de dois glicosímetros veterinários denominados GT1 e GT2. O sistema 
do GT1 mensura a glicemia a partir da oxidação da glicose amostral pela enzima glicose desidrogenase presente na tira teste. Por sua vez, o sistema do GT2 tem a reação enzimática baseada na glicose oxidase presente na tira teste. Ambos GT foram calibrados para fornecerem valores de glicemia em $\mathrm{mg} / \mathrm{dL}$ em amostras de sangue total da espécie canina. Todos os pacientes incluídos no estudo tiveram a glicemia mensurada a partir de amostras de sangue total capilar por cada um dos dois aparelhos avaliados. A cada novo paciente, realizou-se sorteio para determinação da ordem de leitura da amostra capilar pelos GT avaliados. Os procedimentos de coleta e execução imediata da mensuração glicêmica foram realizados pelo mesmo pesquisador, seguindo as instruções de uso dos aparelhos, e respeitando o volume amostral requerido por cada um. ${ }^{(7,14)}$

As amostras capilares foram obtidas a partir da punção de vasos capilares na face interna do pavilhão auricular dos pacientes com auxílio de dispositivo lancetador AlphaTrak ${ }^{\circledR}$ (Abbott Laboratories Ltd., Maidenhead, Inglaterra) e lancetas 28 G. Previamente à punção, foi efetuada a assepsia da superfície cutânea com álcool a $70 \%$ e certificou-se que a superfície estivesse devidamente seca. Assim que a amostra de sangue capilar era obtida, era imediatamente colocada em contato com a face absorvente de cada fita-teste dos GT. A ordem de leitura da amostra capilar seguia a ordem previamente definida por sorteio.

As amostras venosas foram obtidas por punção das veias jugulares ou cefálicas dos pacientes selecionados em condições de antissepsia adequadas e com auxílio de material para coleta, seringas $5 \mathrm{~mL}$ com agulhas 22G (BD Plastipack ${ }^{\mathrm{TM}}$, Juiz de Fora, Brasil). Para a mensuração da glicemia pelos MLab de Hexoquinase (Hx) e Glicose Oxidase (GOx), $4 \mathrm{~mL}$ da amostra foram armazenados em tubos adequados para tal volume contendo o inibidor glicolítico fluoreto de sódio (BD Vacutainer ${ }^{\circledR}$ Fluoreto/EDTA, Juiz de Fora, Brasil). As amostras coletadas para realização de exames de hemograma e análises bioquímicas foram armazenadas em microtubo para hemograma de $0,5 \mathrm{~mL}$ (Vacuplast, Taizhou, China) e em tubo de $4 \mathrm{~mL}$ para coleta de soro (BD Vacutainer®, Juiz de Fora, Brasil). As amostras foram processadas, respectivamente, nos aparelhos semiautomáticos para hematologia CELM CC 530 (Barueri, Brasil) e Cobas-Mira Plus TM (Roche Diagnostics, Indianapolis, Estados Unidos).

\section{Análise dos Dados}

A comparação dos resultados obtidos de cada GT com os do respectivo método laboratorial de referência foi apresentada em gráficos de diferença considerando os intervalos de aceitação de qualidade requeridos pela normativa ISO 15197:2013(11) e conforme descrito por Petersen et al. ${ }^{(15)}$ Para cada comparação entre GT e MLab foram calculados os respectivos coeficientes de correlação, desvios padrão e intervalos de confiança das diferenças. Os gráficos foram confeccionados utilizando-se o aplicativo Analyse-it. As GAE foram elaboradas conforme os limites definidos por Parkes et al. ${ }^{(12)}$ e PFÜTZNER et al. ${ }^{(16)}$ e sua confecção também foi efetuada com o programa Excel versão 2017. Os valores de glicemia mensurados pelos glicosímetros foram comparados a partir da glicemia total capilar e da glicemia estimada para plasma equivalente, conforme proposto por Gerber e Freeman. ${ }^{(8)}$

\section{Resultados}

Foram coletadas amostras de 104 cães, sendo quatro excluídas por não atenderem a pelo menos um dos critérios de inclusão de amostras descritos anteriormente. Em referência ao gênero, 41 pacientes eram machos e 59 fêmeas, destas, sete $(11,86 \%$ do total de fêmeas) manifestavam sinais de cio e $31(52,54 \%)$ eram pan-histerectomizadas. Quanto às idades foi verificada variação entre sete meses e 19 anos. Ao momento das coletas capilares e venosas 42 pacientes estavam sob jejum alimentar maior do que oito horas.

Dentre as 100 amostras, 27 foram coletadas de veias cefálicas e 73 de veias jugulares. De acordo com a ordem definida por sorteio para cada paciente, 58 amostras fossem avaliadas primeiramente pelo GT1 e 42 amostras primeiramente pelo GT2.

Foram usadas 315 fitas teste, 162 fitas do sistema GT1 e 142 fitas do sistema GT2, sendo que todas as fitas usadas em cada GT pertenciam ao mesmo lote de fabricação. Onze pacientes precisaram de mais de uma fita teste em ao menos um dos GT avaliados para a mensuração da glicemia capilar.

$\mathrm{Na}$ comparação entre o GT1 em sangue total capilar e o MLab da Hx (Figura 1) as diferenças tiveram a média de $25,90 \mathrm{mg} / \mathrm{dL}$, desvio padrão de 2,55 e intervalo de confiança de 20,82 a 30,98 . O coeficiente de correlação foi de 0,727 , com $26 \%$ das amostras dentro dos limites de variação das diferenças propostos pela ISO 15197:2013. Por sua vez, quando 
o método do GT1 foi comparado ao mesmo MLab da Hx em valores de glicemia estimados para plasma equivalente, houve a diferença média $10,89 \mathrm{mg} / \mathrm{dL}$, desvio padrão de 0,2636 , intervalo de confiança de 9,3654 e 10,4118 (Figura 2). A correlação foi de $r=$ 0,996 e $99 \%$ das amostras atenderam aos limites de diferença impostos pela ISO 15197:2013.

Figura 1 - Gráfico de diferença entre GT1 em sangue total capilar e o MLab Hx em plasma.

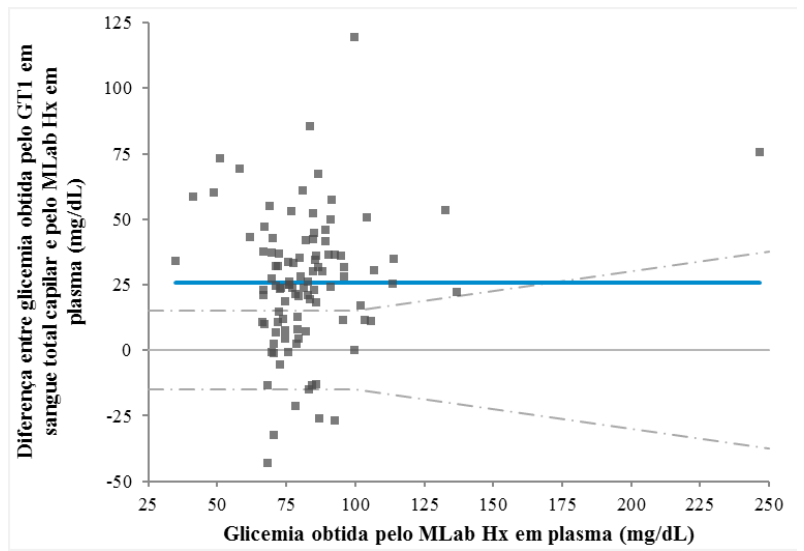

Fonte: Petersen et al. ${ }^{(15)}$

O eixo $\mathrm{x}$ apresenta os valores absolutos de glicemia mensurados pelo MLab da Hx e o eixo y apresenta a diferença entre os valores absolutos de glicemia mensurados pelo GT1 em amostras de sangue total capilar e os mensurados pelo MLab Hx. A diferença média entre os métodos está representada pelas linhas azuis e foi de $25,9 \mathrm{mg} / \mathrm{dL}$. As linhas pontilhadas representam os limites de $\pm 15 \mathrm{mg} / \mathrm{dL}$, para valores de glicemia até $100 \mathrm{mg} / \mathrm{dL}$ e de $15 \%$ para glicemia acima de $100 \mathrm{mg} / \mathrm{dL}$. Belo Horizonte, 2016.
Figura 2 - Gráfico de diferença entre GT1 em plasma equivalente e o MLab da Hx.

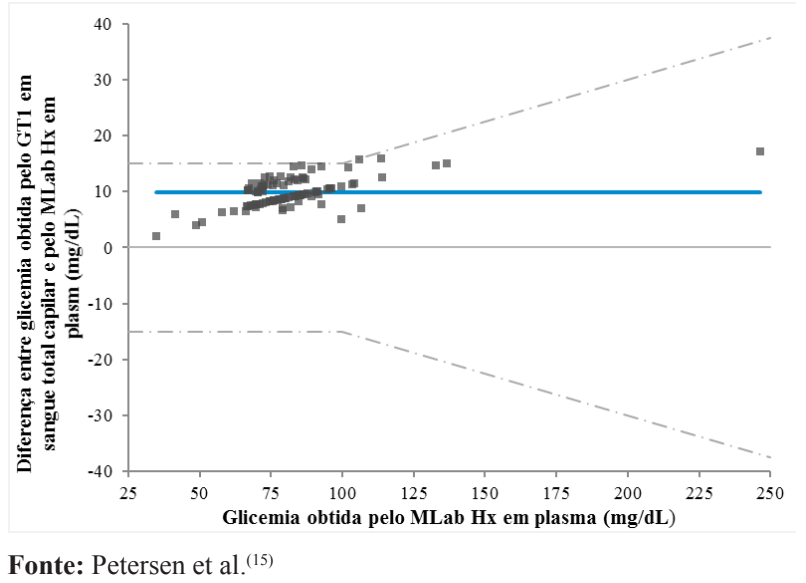

O eixo $\mathrm{x}$ apresenta os valores absolutos de glicemia mensurados pelo MLab da Hx e o eixo y apresenta a diferença entre os valores absolutos de glicemia mensurados pelo GT1 após estimativa para plasma equivalente e os mensurados pelo mesmo MLab da Hx. A diferença média entre os métodos está representada pelas linhas azuis de 10,89 mg/dL. As linhas pontilhadas representam os limites de $\pm 15 \mathrm{mg} /$ $\mathrm{dL}$, para valores de glicemia até $100 \mathrm{mg} / \mathrm{dL}$ e de $15 \%$ para glicemia acima de $100 \mathrm{mg} / \mathrm{dL}$. Belo Horizonte, 2016.

$\mathrm{Na}$ análise das diferenças entre o método GT2 em sangue total capilar e o GOx, foi encontrada média das diferenças de $10,58 \mathrm{mg} / \mathrm{dL}$, desvio padrão de 3,7011 , intervalo de confiança de 3,281 a 17,9256. O coeficiente de correlação entre esses métodos foi de 0,547 e somente $22 \%$ das amostras atenderam aos intervalos de variação de diferenças proposto pela ISO 15197:2013 (Figura 3). Na comparação entre GT2 em plasma equivalente e o MLab GOx, a média das diferenças foi de $9,7029 \mathrm{mg} / \mathrm{dL}$, desvio padrão foi de 0,2724 e intervalo de confiança foi de 9,1623 e 10,2434 . O coeficiente de correlação entre os métodos foi de 0,99 e $99 \%$ das amostras atenderam aos limites impostos pela ISO 15197:2013 (Figura 4). 
Figura 3 - Gráfico de diferença entre GT2 em sangue total capilar e o MLab da GOx.

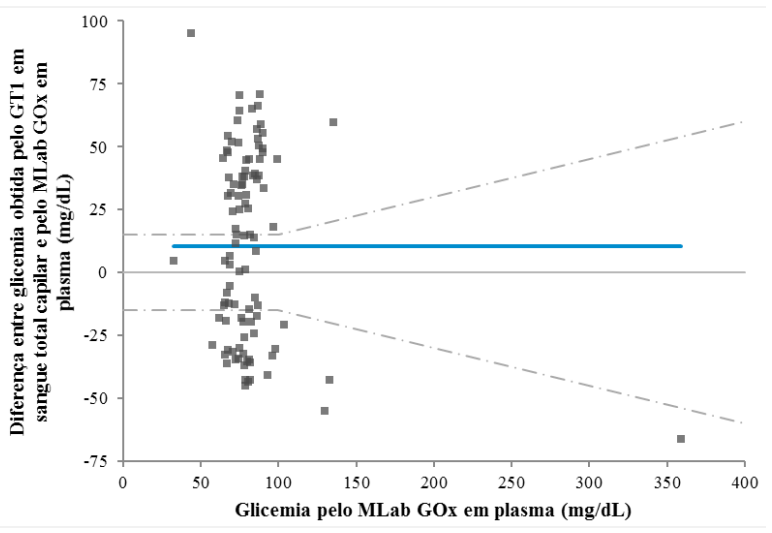

Fonte: Petersen et al. ${ }^{(15)}$

O eixo $\mathrm{x}$ apresenta os valores absolutos de glicemia mensurados pelo MLab da GOx e o eixo y apresenta a diferença entre os valores absolutos de glicemia mensurados pelo GT2 em amostras de sangue total capilar e os mensurados pelo mesmo MLab da GOx. A diferença média entre os métodos está representada pelas linhas azuis e foi de $10,58 \mathrm{mg} / \mathrm{dL}$. As linhas pontilhadas representam os limites de $\pm 15 \mathrm{mg} / \mathrm{dL}$, para valores de glicemia até $100 \mathrm{mg} / \mathrm{dL}$ e de $15 \%$ para glicemia acima de $100 \mathrm{mg} / \mathrm{dL}$. Belo Horizonte, 2016.

Figura 4 - Gráfico de diferença entre GT2 em plasma equivalente e o MLab da GOx.

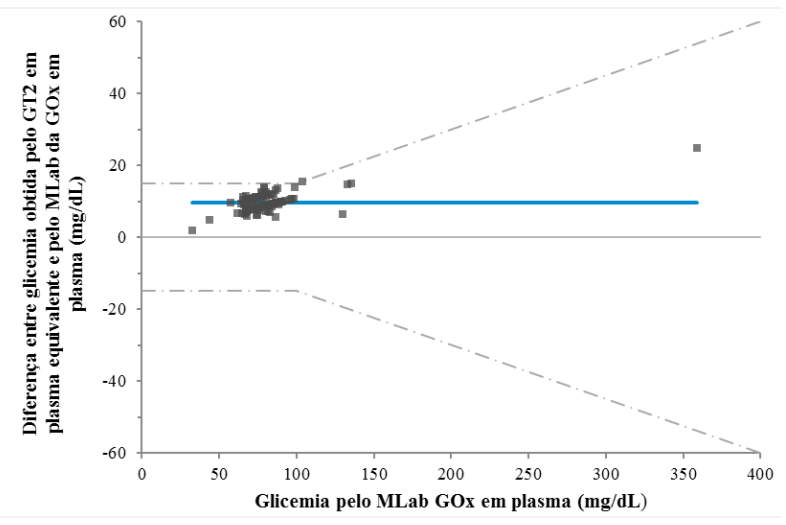

Fonte: Petersen et al. ${ }^{(15)}$

O eixo $\mathrm{x}$ apresenta os valores absolutos de glicemia mensurados pelo MLab da GOx e o eixo y apresenta a diferença entre os valores absolutos de glicemia mensurados pelo GT2 após estimativa para plasma equivalente e os mensurados pelo mesmo MLab da GOx. A diferença média entre os métodos está representada pelas linhas azuis e foi de $9,7029 \mathrm{mg} / \mathrm{dL}$. As linhas pontilhadas representam os limites de $\pm 15 \mathrm{mg} / \mathrm{dL}$, para valores de glicemia até $100 \mathrm{mg} / \mathrm{dL}$ e de $15 \%$ para glicemia acima de $100 \mathrm{mg} / \mathrm{dL}$. Belo Horizonte, 2016.

A análise pela grade de análise de erros para avaliação da acurácia clínica do GT1 em comparação ao MLab da Hx teve $43 \%$ dos resultados recaindo sobre a zona A, $44 \%$ sobre a zona B e $13 \%$ e $1 \%$, respectivamente sobre as zonas $\mathrm{C}$ e D, conforme apresentado na Figura 5. Sendo assim, $87 \%$ das amostras recaíram sobre as zonas A e B, que são consideradas clinicamente acuradas pela ISO 15197:2013. O intervalo de confiança da média foi de 97,7728 .

Figura 5 - Grade de análise de erros de Parkes para glicemia mensurada pelo GT1.

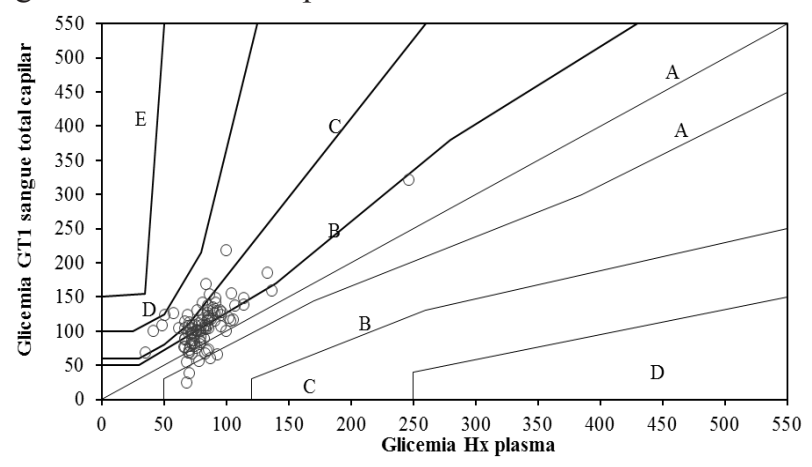

Fonte: Parkes et al. ${ }^{(12)}$

Os valores foram mensurados a partir de amostras de sangue total capilar de e amostras plasmática. No eixo x estão apresentados os valores de glicemia pelo MLab Hx e no eixo y estão apresentados os valores de glicemia pelo GT1. Todos os valores de glicemia estão em mg/dL. Belo Horizonte, 2016.

Da avaliação do GT2 pela GAE, que teve como parâmetro o MLab da GOx, 33\% das amostras recaíram sobre a zona A, $53 \%$ sobre a zona $\mathrm{B}, 13 \%$ sobre a C e $1 \%$ sobre a D. O total de $86 \%$ das amostras alcançou as zonas consideradas acuradas pela ISO 15197:2013, conforme apresentado na Figura 6. Neste caso, o intervalo de confiança foi de 101,6719. 
Figura 6 - Grade de Análise de Erros de Parkes para glicemia mensurada pelo GT2.

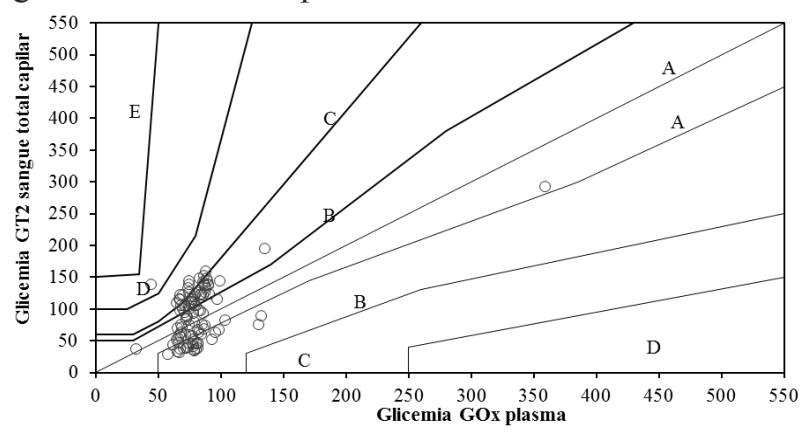

Fonte: Parkes et al. ${ }^{(12)}$

Os valores foram mensurados a partir de amostras de sangue total capilar e de amostras plasmáticas. No eixo x estão apresentados os valores de glicemia pelo MLab GOx e no eixo y estão apresentados os valores de glicemia pelo GT2. Todos os valores de glicemia estão em mg/dL. Belo Horizonte, 2016.

\section{Discussão}

Durante a execução deste estudo foi observada possível interferência do usuário sobre a acurácia dos testes, no que se refere ao manuseamento do aparelho e à coleta da amostra capilar. Pois dentre os quatorze pacientes que requereram mais de uma fita teste para a mensuração da glicemia capilar, nove estavam entre os 50 primeiros pacientes incluídos. Ou seja, existe a possibilidade de que a prática do usuário para o manejo dos GT possa prejudicar a eficiência dos testes. A normativa ISO de 2013 inclui algumas recomendações para avaliação do grau de interferência do manuseamento sobre a acurácia clínica dos aparelhos.

$\mathrm{O}$ método adotado neste estudo controlou alguns fatores reconhecidos como fontes de erro em potencial, entre eles alguns que foram propostos pelas respectivas bulas dos GT abordados. No entanto, a abordagem dos GT buscou explorar seu uso ambulatorial, dentro dos padrões pretendidos por cada fabricante, sendo assim, algumas fontes de interferência foram incorporadas simulando uma situação de uso rotineiro. Outros autores abordaram anteriormente o uso domiciliar e não profissional dos GT em pequenos animais através da aplicação de questionários, reforçando a importância de que tais avaliações sejam procedidas em medicina veterinária. $^{(17)}$
Para abordagem do parâmetro de acurácia dos aparelhos, os resultados de glicemia fornecidos por eles foram comparados com dois MLab, Hx e GOx. A acurácia exibida por cada GT, apesar dos erros analíticos intrínsecos, foi quantificada através dos coeficientes de variação que definem os limites de variação dos resultados do método, de modo que torna-se possível compará-los com critérios propostos, como os da ISO 15179:2013. ${ }^{(18)}$

Sob o modelo dos gráficos de diferença, a decisão sobre a aceitação da variação entre a glicemia mensurada pelos dois métodos tem como critério fundamental a dispersão dessa diferença em relação a glicemia mensurada pelo MLab, sendo ambas avaliadas quanto a seus valores absolutos. Neste caso, o julgamento da aceitação da validade do método do GT é baseada em metodologia com consagrada acurácia para mensuração do mesmo analito. ${ }^{(15)}$ Autores de referência destacam que método eleito como referência tem de ser adequado quanto ao tipo de amostra, à equivalência das reações enzimáticas e ao controle das condições ambientais para certificação de sua acurácia e sensibilidade. ${ }^{(15,19)}$

A acurácia reduzida exibida pelos GT quando avaliados em sangue capilar total, tem como ponto crítico a comparação de valores de glicemia mensurados a partir de amostras diferentes. ${ }^{(13)}$ Apesar de a glicemia mensurada por cada GT ter sido comparada a glicemia mensurada pelo MLab mais adequado, cada um desses métodos foi abordado a partir de um tipo de amostra: os do GT pelo sangue total capilar e os do MLab pelo plasma venoso. Como a distribuição da glicose difere entre tais amostras devido à concentração de água e solutos, a comparação entre essas duas abordagens não considera duas condições hematimétricas equivalentes. A configuração da diferença da glicemia entre sangue capilar e venoso é ainda mais pronunciada no período pós-prandial, quando o sangue capilar pode alcançar concentração de glicose até $25 \%$ maior que o venoso. ${ }^{(20)}$

A normativa ISO 15197:2013 sugere que a acurácia seja avaliada pelo modelo dos gráficos das diferenças que permite a imposição dos critérios de variação das diferenças entre a glicemia mensurada pelo GT e a mensurada pelo MLab. A normativa postula que tal diferença pode variar $\pm 15 \mathrm{mg} / \mathrm{dL}$ em amostras cujo MLab tenha mensurado até $100 \mathrm{mg} / \mathrm{dL}$, e em $\pm 15 \%$ em amostras cujo MLab tenha mensurado glicemia acima de $100 \mathrm{mg} / \mathrm{dL}$. A apresentação visual - as linhas horizontais pontilhadas - permite o julgamento para aceitação do GT avaliado caso seus resultados recaiam sobre os específicos limites de variação (Figuras 1 e 2). De acordo com a ISO 15197:2013, o GT atende ao critério de acurácia caso 
95\% dos resultados forem distribuídos entre os limites definidos, ou seja, o GT será acurado na medida em que tenha identidade com o MLab.

No entanto, a concentração da glicose no sangue total é uma variável capaz de alterar a viscosidade da amostra e, portanto, pode interferir na disponibilidade de glicose para reação na fita teste, comprometendo a acurácia do sistema. ${ }^{(21)}$ Portanto, o desempenho do GT pode ser distinto em função da magnitude da glicemia. Posto assim, os limites impostos pela ISO 15197:2013 variam de valores absolutos a percentuais em função da glicemia mensurada pelo MLab.

A padronização da acurácia dos GT pelos gráficos de diferença garante a avaliação qualitativa da performance do aparelho, uma vez que procede as comparações incluindo os erros que podem interferir no método. Contudo, como não se procede a identificação e mensuração de tais erros para cada um dos métodos comparados, a imprecisão de cada um deles se reflete na média das diferenças. Na comparação dos GT em plasma equivalente com MLab, foram encontradas as médias de diferença de: 9,88 mg/dL entre Hx e GT1 e de 9,7 mg/dL entre GOx e GT2 (Figuras 1 e 3). Com relação aos desvios padrões das diferenças, citado pela literatura como sendo o fator que estima o erro inerente aos métodos, foram menores nas comparações nas quais se identificou maior identidade entre GT e MLab. ${ }^{(15)}$

Finalmente, as comparações que identificaram identidade entre GT e MLab foram aquelas cujos GT tiveram $\geq 99 \%$ de amostras dentro do intervalo de acurácia pretendido pela ISO15197:2013, a saber, GT1 e GT2, sendo ambos para plasma equivalente. Tais comparações constataram a acurácia pretendida pela ISO15197:2013 foram aquelas nas quais as variáveis de avaliação eram controladas de modo a manter e garantir a comparação de semelhantes para tipo de amostra (plasma vs. plasma equivalente) e enzimas do sistema de mensuração da glicemia (Hx e GOx). ${ }^{(13,22)}$.

A análise pelas GAE de Parkes et al. ${ }^{(12)}$ é uma avaliação proposta pela ISO 15197:2013 que permite a avaliação da acurácia dos GT apesar das interferências de erros analíticos inerentes tanto ao GT quanto ao MLab usado como referência. ${ }^{(11,16)}$

Por sua vez, as GAE avaliaram a interferência que os erros das mensurações fornecidos pelos GT apresentariam caso decisões clínicas fossem baseadas em tais testes. Assim, como os gráficos de diferença, as GAE abordam dados pareados para comparar os dois métodos, respeitando o mesmo sistema enzimático para as comparações. No entanto, as GAE abordam valores absolutos ao invés da diferença dos resultados. ${ }^{(12,16)}$ Os dados são distribuídos nas zonas pré-determinadas em função da discrepância entre os resultados fornecidos por cada método, zonas nomeadas de A a Z correspondentes a graus crescentes de comprometimento da conduta clínica em função dos erros. A plotagem nas zonas parte da referência da zona A, sobre a qual recaem valores fornecidos pelos GT que variam em até $20 \%$ em relação aos fornecidos pelo MLab. A GAE quantifica a acurácia clínica do método avaliado, uma vez que permite associar dados referentes a intercorrências clínicas em dados numéricos apresentados em gráfico de dispersão. ${ }^{(16,23)}$

No presente estudo, as GAE foram elaboradas com dados de glicemia conforme são fornecidos pelos GT, de modo que representam a glicemia capilar total. A acurácia clínica apresentada através dessas comparações, de menos de aproximadamente $85 \%$ para ambos GT, é considerada insuficiente para os parâmetros da ISO15197:2013 e pode ser explicada por não ter sido respeitado o critério que sugere o uso do mesmo tipo de amostra nesse tipo de avaliação. ${ }^{(24-25)} \mathrm{A}$ escolha por avaliar a acurácia clínica com base exclusivamente nos resultados de GT para glicemia capilar foi feita para garantir a simulação de condições da rotina de uso dos aparelhos. ${ }^{(13)}$

Neste ponto é interessante confrontar a acurácia analítica apresentada pelos gráficos de diferença com a acurácia clínica apresentada pelas GAE. Quando os GT foram avaliados pelas amostras de sangue total capilar, ambos não alcançaram os limites de acurácia pretendidos pela normativa ISO15197:2013, 95\% e 99\% das amostras para os gráficos de diferença e para as GAE, respectivamente. E a acurácia analítica foi ainda menor em comparação à acurácia clínica, para os dois GT. Uma observação que está de acordo com as pesquisas de Pfützner et al.(16) é de que a acurácia analítica é mais estreita em relação à acurácia clínica, pois é susceptível à interferência de fatores envolvidos em todo protocolo de avaliação, desde a coleta das amostras até os possíveis erros dos MLab usados como referência (Figuras 1 e 3 para os resultados de GT1, e Figuras 2 e 4 para os resultados de GT2). Os mesmos autores esclarecem que a avaliação da acurácia clínica a partir das GAE permite a projeção das consequências clínicas da ocorrência dos erros de mensuração de glicemia pelos GT, apesar de todas as interferências que podem estar envolvidas nos processos de teste.

O estudo dos erros analíticos de cada de GT é uma avaliação que engloba quatro categorias de fontes de erro: a imprecisão da análise, as interferências relativas à randomização dos pacientes, e, os erros relativos aos 
métodos de mensuração e ao protocolo definido ${ }^{(9,13)}$ As referências da área propõem que a abordagem de tais fontes de erro pode ser didaticamente separada de acordo com as etapas de teste sobre as quais incidem, para que a interferência de cada fator possa ser determinado, bem como sejam cuidadosamente controladas em estudos de avaliação de acurácia, ${ }^{(26,27)}$ que, no entanto, não foi objeto deste estudo.

Sob a perspectiva da avaliação dos GT para incrementar a confiabilidade em seu uso na rotina clínica veterinária, as GAE permitiram a representação clara e direta que possível inacurácia apresentada pelos aparelhos pode provocar sobre as condutas terapêuticas. Enquanto estratégia de avaliação, as GAE estabelecem um foco mais objetivo e usual, uma vez que avaliam o aparelho com as interferências que impreterivelmente incidem sobre os teste rápidos de mensuração da glicemia. ${ }^{(8,12)}$

\section{Conclusão}

Enquanto ferramentas para monitoramento do perfil glicêmico imediato, GT 1 e 2 não apresentaram desempenho satisfatório de acordo com os parâmetros de acurácia analítica e clínica propostos pela ISO 15197:2013. Invariavelmente, a baixa acurácia demonstrada prejudica a confiabilidade do clínico na adoção de tais ferramentas para rotina de monitoramento glicêmico dos pacientes. Discretas deturpações dos valores reais de glicemia fornecidas por um GT podem implicar em graves erros nas condutas terapêuticas e agravar um quadro de disglicemia, colocando em risco a vida do paciente.

\section{Agradecimentos}

Os autores agradecem à Escola de Veterinária da Universidade Federal de Minas Gerais, aos colegas médicos veterinários e aos acadêmicos que participaram da execução deste projeto. Agradecimento especial ao M.V. prof. Dr. Állan Gomes Pöppl pela contribuição na elaboração da grade de análise de erros.

\section{Referências}

1 Cheng WJ, Lin CW, Wu TG, Su CS, Hsieh MS. Calibration of glucose oxidase-based test strips for capillary blood measurement with oxygen saturated venous blood samples. Clin Chim Acta. 2013 Jan;415:152-157. doi: 10.1016/j.cca.2012.10.033

2 Wahl HG. How accurately do we measure blood glucose levels in intensive care unit (ICU) patients? Best Pract Res Clin Anesthesiol. 2009;23(4):387400. doi: 10.1016/j.bpa.2009.09.003

3 Kley S, Casella M, Reusch CE. Evaluation of longterm monitoring of blood glucose concentrations in cats with diabetes mellitus: 26 cases (1999-2002). J Am Vet Med Assoc. 2004 Jul;225(2):261-266

4 Hermayer KL, Loftley AS, Reddy S, Narla SN, Epps NA, Zhu Y. Challenges of inpatient blood glucose monitoring: standards, methods, and devices to measure blood glucose. Curr Diab Rep. 2015 Mar;15(3):10. doi: 10.1007/s11892-015-0582-9

5 Bluwol K, Duarte R, Lustosa MD, Simões DMN, Kogika MM. Evaluation of two portable meters for blood glucose measurements in dogs. Arq. Bras. Med. Vet. Zootec. 2007 Dec;56(6):1408-1411. doi: 10.1590/S0102-09352007000600009

6 Freckmann G, Baumstark A, Jendrike N, Zschornak E, Kocher S, Tshiananga J, et al. System accuracy evaluation of 27 blood glucose monitoring systems according to DIN EN ISO 15197. Diabetes Technol Ther. 2010 Mar;12(3):221-31. doi: 10.1089/ dia.2009.0128

7 Brito-Casillas Y, Figueirinhas P, Wiebe JC, LópezRíos L, Pérez-Barreto D, Melián C et al. ISO-Based Assessment of Accuracy and Precision of Glucose Meters in Dogs. J Vet Intern Med. 2014 SepOut;28(5):1405-13. doi: 10.1111/jvim.12397

8 Gerber KL, Freeman KP. ASVCP guidelines: quality assurance for portable blood glucose meter (glucometer) use in veterinary medicine. Vet Clin Pathol. 2016 Mar;45(1):10-27. doi: 10.1111/ vcp. 12310 
9 Krouwer JS, Cembrowski GS. A review of standards and statistics used to describe blood glucose monitor performance. J Diabetes Sci Technol. 2010 Jan;4(1):75-83

10 Schnell O, Hinzmann R, Kulzer B, Freckmann G, Erbach M, Lodwig V, et al. Assessing the analytical performance of systems for self-monitoring of blood glucose: concepts of performance evaluation and definition of metrological key terms. J Diabetes Sci Technol. 2013 Nov;7(6):1585-94. doi: $10.1177 / 193229681300700619$

11 International Organization for Standadization. ISO 15197:2013(en) In vitro diagnostic test systems: requirements for blood-glucose monitoring systems for self-testing in managing diabetes mellitus [Internet] [acesso 2016 Nov. 28]. Disponível em: https://www.iso.org/obp/ui/\#iso:std:iso:15197:ed2:v1:en

12 Parkes JL, Slatin SL, Pardo S, Ginsberg BH. A new consensus error grid to evaluate the clinical significance of inaccuracies in the measurement of blood glucose. Diabetes Care. 2000;23(8):1143-1148.

13 Thorpe GH. Assessing the quality of publications evaluating the accuracy of blood glucose monitoring systems. Diabetes Technol Ther. 2013 Mar;15(3):2539.

14 Ferreira PA, Mueller EM, Fischer EC, Tillmann MT, Peres W, Nobre MO. Glicemia do sangue capilar e venoso de cães saudáveis: mensuração por método eletroquímico versus enzimático laboratorial. Semina: Ciênc Agrár. 2013;34(3):128792.

15 Petersen PH, Stöckl D, Blaabjerg O, Pedersen B, Bikermose E, Thienpont L et al. Graphical interpretation of analytical data from comparison of a field method with a reference method by use of difference plots. Clin Chem. 1997 Nov;43(11):203946

16 Pfützner A, Klonoff DC, Pardo S, Parkes JL. Technical aspects of the Parkes error grid. J Diabetes Sci Technol. 2013 Sep;7(5):1275-81. doi: $10.1177 / 193229681300700517$
17 Van De Maele I, Rogier N, Daminet S. Retrospective study of owners' perception on home monitoring of blood glucose in diabetic dogs and cats. Can Vet J. 2005 Aug;46(8):718-23.

18 ROSS, J. W.; FRASER, M. D. Analytical goals developed from the inherent error of medical tests. Clin Chem. 1993;39(7):1481-94.

19 Westgard J. Points of care in using statistics in method comparison studies. Clin Chem. 1998;(11):2240-2.

20 Tonyushkina K, Nichols JH. Glucose meters: a review of technical challenges to obtaining accurate results. J Diabetes Sci Technol. 2009 Jul;3(4):971-80

21 Çinar Y, Şenyol AM, Duman K. Blood viscosity and blood pressure: role of temperature and hyperglycemia. Am J Hypertens. 2001 May;14(5):433-8, 2001.

22 Freckmann G, Schmid C, Baumstark A, Rutschmann M, Haug C, Heinemann L. Analytical performance requirements for systems for self-monitoring of blood glucose with focus on system accuracy: relevant differences among ISO 15197:2003, ISO 15197:2013, and current FDA recommendations. J Diabetes Sci Technol. 2015 Jul;9(4):885-94, 2015.

23 Domori A, Sunahara A, Tateno M, Miyama TS, Setogushi A, Endo Y. The clinical utility of two human portable blood glucose meters in canine and feline practice. Vet Clin Pathol. 2013 Mar; 43,(1):5562. doi: $10.1111 /$ vcp. 12115

24 Link M, Pleus S, Schmid C, Freckmann G, Baumstark A, Stolberg E, et al. Accuracy evaluation of three systems for self-monitoring of blood glucose with three different test strip lots following ISO 15197. J Diabetes Sci Technol. 2014 Mar;8(2):422-4. doi: $10.1177 / 1932296813518859$

25 Nelson RW. Canine diabetes mellitus. In: Ettinger SJ, Feldman EC (Eds.). Textbook of veterinary internal medicine. $7^{\text {th }}$ ed. Saint Louis: Elsevier Saunders; 2010. p. 2218

26 Sirois M. Clinical chemistry. In: Hendrix CM, Sirois M. (Eds.). Laboratory procedures for veterinary technicians. $5^{\text {th }}$ ed. Canada: Mosby Elsevier; 2007. p. 416 
27 Sacks DB, Arnold M, Bakris GL, Bruns DE, Horvath AR, Kirkman MS et al. Guidelines and recommendations for laboratory analysis in the diagnosis and management of diabetes mellitus. Diabetes Care. 2011 Jun;34(6):61-9. doi: 10.2337/ dc11-9998 